\title{
Higher Order Mechanical and Electric Fields for Arbitrarily Oriented Crack with the Physical Weak-Discontinuity
}

\author{
Yao Dai ${ }^{1, a}$, Xiao Chong ${ }^{2, b}$, Yumeng Shi $i^{3, b}$ and Jincai Huang ${ }^{4, a}$ \\ ${ }^{1}$ Academy of Armored Force Engineering, Beijing, 100072, China \\ ${ }^{2}$ Changping School of Noncommissioned Officer, Academy of Equipment, Beijing, 101416, China \\ ${ }^{3}$ China Academy of Launch Vehicle Technology \\ ${ }^{4}$ China National Machinery Industry Corporation, Beijing \\ adai_yao@sina.com, b chongxiao2005@163.com
}

Keywords: crack tip fields, piezoelectric materials, arbitrarily oriented crack, weak-discontinuity

\begin{abstract}
The higher order crack-tip fields for anti-plane crack oblique to the interface between functionally graded piezoelectric materials (FGPMs) and homogeneous piezoelectric materials(HPMs) are presented. The crack is oriented in arbitrary direction. The crack surfaces are assumed to be electrically impermeable. The material properties of FGPMs are assumed to be exponential functions with their gradient direction perpendicular to the interface. By using the eigen-expansion method, the high order crack tip stress and electric displacement fields are obtained. The analytic expressions of the stress intensity factors and the electric displacement intensity factors are derived.
\end{abstract}

\section{Introduction}

Piezoelectric ceramics are extensively used in design of various electromechanical devices such as transducers, sensors, and actuators. The fracture mechanics of piezoelectric materials has received much interest over a decade. A lot of literature can be found for theoretical predications and experimental investigations of fracture parameters, i.e., field intensity factors and energy release rate. $\mathrm{Li}$ and Weng[1] considered the static anti-plane problem of a finite crack in a functionally graded piezoelectric material strip. It is found that the singular behaviors of both stress and electric displacement are the same as those in a homogeneous piezoelectric material. Ing and Chen [2] investigated the transient response of an interfacial crack between two functionally graded piezoelectric strips. Singh[3] studied the problem of an antiplane crack situated in the interface of two bonded dissimilar graded piezoelectric half-spaces under the permeable crack assumption. Yeh and Chue [4] investigated the anti-plane crack problem of a FGPM cracked strip bonded to an FGPM cracked half-plane. Han[5] calculated the plane electro-elastic fields in piezoelectric materials with multiple cracks. All of the above mentioned works are solved the singular part of crack tip fields by Fourier transforms and singular integral equations. The higher order crack tip fields for arbitrarily oriented crack oblique to the interface of piezoelectric materials has not been studied. The higher order mechanical and electric fields and explicit expressions of intensity factors will be presented.

\section{Basic equations}

Fig. 1 shows the configuration of FGPMs bonded to HPMs with an arbitrarily oriented crack. Regions I, II and III are defined as materials 1, 2 and 3, respectively. Consider an impermeable crack of length $2 L$ oblique to the interface subjected to anti-plane shear loads and the in-plane electric displacements. The poling directions of piezoelectric materials are orientated along $z$-axis. The present work employs exponential function to describe the continuous variations of material properties, 


$$
\begin{gathered}
c_{44}^{(2)}=c_{44}^{(1)} f(r, \theta), \quad e_{15}^{(2)}=e_{15}^{(1)} g(r, \theta), \quad \varepsilon_{11}^{(2)}=\varepsilon_{11}^{(1)} j(r, \theta) \\
f(r, \theta)=e^{\beta_{1} r \sin (\theta+\alpha)}, \quad g(r, \theta)=e^{\beta_{2} r \sin (\theta+\alpha)}, \quad j(r, \theta)=e^{\beta_{3} r \sin (\theta+\alpha)}
\end{gathered}
$$

where $c_{44}^{(1)}$ is the shear modulus, $e_{15}^{(1)}$ is the piezoelectric coefficient, $\varepsilon_{11}^{(1)}$ is the dielectric parameter of the HPM.

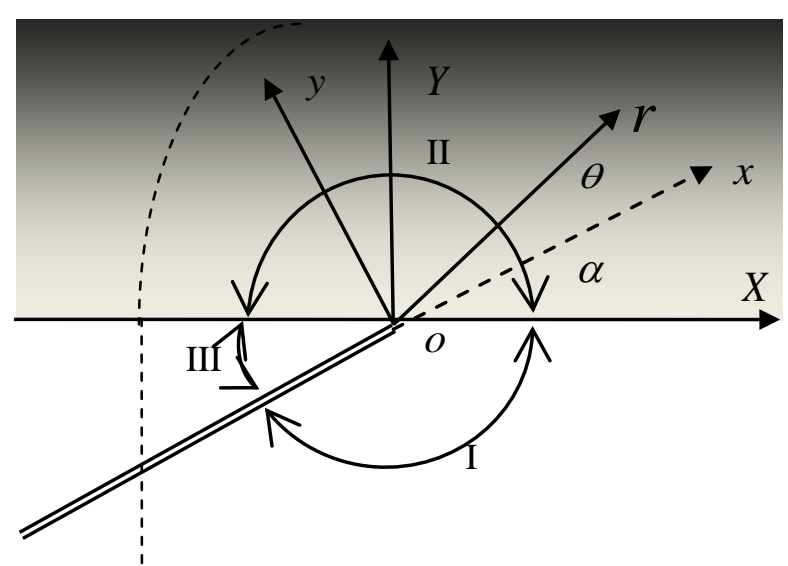

Fig.1 Anti-plạine oblique crack in piezoelectric materials

The governing equations can be written as

$$
\left\{\begin{array}{l}
c_{44}^{(k)} \nabla^{2} w+\frac{\partial c_{44}^{(k)}}{\partial r} \frac{\partial w}{\partial r}+\frac{\partial c_{44}^{(k)}}{r^{2} \partial \theta} \frac{\partial w}{\partial \theta}+e_{15}^{(k)} \nabla^{2} \phi+\frac{\partial e_{15}^{(k)}}{\partial r} \frac{\partial \phi}{\partial r}+\frac{\partial e_{15}^{(k)}}{r^{2} \partial \theta} \frac{\partial \phi}{\partial \theta}=0 \\
e_{15}^{(k)} \nabla^{2} w+\frac{\partial e_{15}^{(k)}}{\partial r} \frac{\partial w}{\partial r}+\frac{\partial e_{15}^{(k)}}{r^{2} \partial \theta} \frac{\partial w}{\partial \theta}-\varepsilon_{11}^{(k)} \nabla^{2} \phi-\frac{\partial \varepsilon_{11}^{(k)}}{\partial r} \frac{\partial \phi}{\partial r}-\frac{\partial \varepsilon_{11}^{(k)}}{r^{2} \partial \theta} \frac{\partial \phi}{\partial \theta}=0
\end{array}\right.
$$

where $\nabla^{2}=\frac{\partial^{2}}{\partial r^{2}}+\frac{1}{r} \frac{\partial}{\partial r}+\frac{1}{r^{2}} \frac{\partial^{2}}{\partial \theta^{2}}$ is the two-dimensional Laplace operator.

\section{The higher order crack-tip field}

The displacement component $w$ and the electric potential $\phi$ can be expanded as follows

$$
w^{(k)}=\sum_{i=1}^{\infty} r^{\frac{i}{2}} w_{i}^{(k)}(\theta), \quad \phi^{(k)}=\sum_{i=1}^{\infty} r^{\frac{i}{2}} \phi_{i}^{(k)}(\theta)
$$

where, $w_{i}^{(k)}(\theta)$ and $\phi_{i}^{(k)}(\theta)$ are eigen-functions.

Substitute Eq.(4) into Eq.(3). According to the linear independence of $r^{-3 / 2}, r^{-1}$, $r^{-1 / 2}, \ldots, r^{i / 2-2}, \ldots$, the system of ordinary differential equations are obtained. Solving the system of ordinary differential equations, we can obtain the results

$$
\begin{aligned}
& \left\{\begin{array}{l}
w_{1}^{(2)}(\theta)=B_{11} \sin \frac{\theta}{2}+B_{12} \cos \frac{\theta}{2} \\
\phi_{1}^{(2)}(\theta)=B_{13} \sin \frac{\theta}{2}+B_{14} \cos \frac{\theta}{2}
\end{array}\right. \\
& \left\{\begin{array}{l}
w_{2}^{(2)}(\theta)=B_{22} \sin \theta+B_{21} \cos \theta \\
\phi_{2}^{(2)}(\theta)=B_{24} \sin \theta+B_{23} \cos \theta
\end{array}\right.
\end{aligned}
$$




$$
\left\{\begin{aligned}
& w_{3}^{(2)}(\theta)= B_{31} \sin \frac{3 \theta}{2}+B_{32} \cos \frac{3 \theta}{2}-\frac{\beta_{1} c_{440} \varepsilon_{110}+\beta_{2} e_{150}^{2}}{4\left(c_{440} \varepsilon_{110}+e_{150}^{2}\right)}\left[B_{12} \sin \left(\frac{\theta}{2}+\alpha\right)+B_{11} \cos \left(\frac{\theta}{2}+\alpha\right)\right] \\
&-\frac{e_{150} \varepsilon_{110}\left(\beta_{2}-\beta_{3}\right)}{4\left(c_{440} \varepsilon_{110}+e_{150}^{2}\right)}\left[B_{14} \sin \left(\frac{\theta}{2}+\alpha\right)+B_{13} \cos \left(\frac{\theta}{2}+\alpha\right)\right] \\
& \phi_{3}^{(2)}(\theta)= B_{33} \sin \frac{3 \theta}{2}+B_{34} \cos \frac{3 \theta}{2}-\frac{c_{440} e_{150}\left(\beta_{1}-\beta_{2}\right)}{4\left(c_{440} \varepsilon_{110}+e_{150}^{2}\right)}\left[B_{12} \sin \left(\frac{\theta}{2}+\alpha\right)+B_{11} \cos \left(\frac{\theta}{2}+\alpha\right)\right] \\
&+ \frac{\beta_{2} e_{150}^{2}+\beta_{3} c_{440} \varepsilon_{110}}{4\left(c_{440} \varepsilon_{110}+e_{150}^{2}\right)}\left[B_{14} \sin \left(\frac{\theta}{2}+\alpha\right)+B_{13} \cos \left(\frac{\theta}{2}+\alpha\right)\right] \\
& w_{4}^{(2)}(\theta)= B_{42} \sin 2 \theta+B_{41} \cos 2 \theta-\frac{\beta_{1} c_{440} \varepsilon_{110}+\beta_{2} e_{150}^{2}}{4\left(c_{440} \varepsilon_{110}+e_{150}^{2}\right)}\left(B_{21} \sin \alpha+B_{22} \cos \alpha\right) \\
&- \frac{e_{150} \varepsilon_{110}\left(\beta_{2}-\beta_{3}\right)}{4\left(c_{440} \varepsilon_{110}+e_{150}^{2}\right)}\left(B_{23} \sin \alpha+B_{24} \cos \alpha\right) \\
& \phi_{4}^{(2)}(\theta)=B_{44} \sin 2 \theta+B_{43} \cos 2 \theta-\frac{c_{440} e_{150}\left(\beta_{1}-\beta_{2}\right)}{4\left(c_{440} \varepsilon_{110}+e_{150}^{2}\right)}\left(B_{21} \sin \alpha+B_{22} \cos \alpha\right) \\
&+ \frac{\beta_{2} e_{150}^{2}+\beta_{3} c_{440} \varepsilon_{110}}{4\left(c_{440} \varepsilon_{110}+e_{150}^{2}\right)}\left(B_{23} \sin \alpha+B_{24} \cos \alpha\right) \\
& \mathbf{w}=\sum_{i=1}^{+\infty}\left[r^{i-1 / 2} \mathbf{A}_{2 i-1} \sin \left(i-\frac{1}{2}\right) \theta+r^{i} \mathbf{A}_{2 i} \cos i \theta\right]
\end{aligned}\right.
$$

where $\quad \mathbf{w}=\left[\begin{array}{llll}w^{(1)} & \phi^{(1)} & w^{(3)} & \phi^{(3)}\end{array}\right]^{\mathrm{T}} \quad, \quad \mathbf{A}_{2 i-1}=\left[\begin{array}{lllll}A_{(2 i-1) 1} & A_{(2 i-1) 2} & C_{(2 i-1) 1} & C_{(2 i-1) 2}\end{array}\right]^{\mathrm{T}} \quad$, $\mathbf{A}_{2 i}=\left[\begin{array}{llll}A_{(2 i) 1} & A_{(2 i) 2} & C_{(2 i) 1} & C_{(2 i) 2}\end{array}\right]^{\mathrm{T}}, A_{i j}, C_{i j}$ are the undetermined coefficients.

Substituting Eq. into Eq.(4), the displacement component and the electric potential for the FGPM and HPM regions are obtained. Then the stress and electric displacement fields will be obtained based on constitutive relations as

$$
\left\{\begin{array}{l}
\tau_{x z}^{(k)}=c_{44}^{(k)} w_{, x}^{(k)}+e_{15}^{(k)} \phi_{, x}^{(k)} \\
\tau_{y z}^{(k)}=c_{44}^{(k)} w_{, y}^{(k)}+e_{15}^{(k)} \phi_{, y}^{(k)} \\
D_{x}^{(k)}=e_{15}^{(k)} w_{, x}^{(k)}-\varepsilon_{11}^{(k)} \phi_{, x}^{(k)} \\
D_{y}^{(k)}=e_{15}^{(k)} w_{, y}^{(k)}-\varepsilon_{11}^{(k)} \phi_{, y}^{(k)}
\end{array}\right.
$$

The mechanical and electric conditions must satisfy the following continuous conditions along the interface

$$
\begin{gathered}
\left.\sigma_{z y}^{(1)}\right|_{\theta=-\alpha}=\left.\sigma_{z y}^{(2)}\right|_{\theta=-\alpha} \\
\left.D_{y}^{(1)}\right|_{\theta=-\alpha}=\left.D_{y}^{(2)}\right|_{\theta=-\alpha} \\
\left.\sigma_{z y}^{(2)}\right|_{\theta=\pi-\alpha}=\left.\sigma_{z y}^{(3)}\right|_{\theta=\pi-\alpha} \\
\left.D_{y}^{(2)}\right|_{\theta=\pi-\alpha}=\left.D_{y}^{(3)}\right|_{\theta=\pi-\alpha}
\end{gathered}
$$

Substituting Eq. (10)into Eq.(11), the relations between $A_{i j}, B_{i j}$ and $C_{i j}$ can be obtained 


$$
\begin{aligned}
& A_{11}=B_{11}+B_{12} \tan \frac{\alpha}{2} \\
& A_{12}=B_{13}+B_{14} \tan \frac{\alpha}{2} \\
& A_{21}=B_{21}+B_{22} \cot \alpha \\
& A_{22}=B_{23}+B_{24} \cot \alpha \\
& A_{31}=B_{31}+B_{32} \tan \frac{3 \alpha}{2}+\frac{1}{12\left(c_{440} \varepsilon_{110}+e_{150}^{2}\right) \cos \frac{3 \alpha}{2}}\left[\left(\beta_{1} c_{440} \varepsilon_{110}+\beta_{2} e_{150}^{2}\right)\right. \text {. } \\
& \left.\left(B_{11} \sin \frac{\alpha}{2}-B_{12} \cos \frac{\alpha}{2}\right)+\left(\beta_{2}-\beta_{3}\right) e_{150} \varepsilon_{110}\left(B_{13} \sin \frac{\alpha}{2}-B_{14} \cos \frac{\alpha}{2}\right)\right] \\
& A_{32}=B_{33}+B_{34} \tan \frac{3 \alpha}{2}+\frac{1}{12\left(c_{440} \varepsilon_{110}+e_{150}^{2}\right) \cos \frac{3 \alpha}{2}}\left[( \beta _ { 1 } - \beta _ { 2 } ) c _ { 4 4 0 } e _ { 1 5 0 } \left(B_{11}\right.\right. \\
& \left.\left.\sin \frac{\alpha}{2}-B_{12} \cos \frac{\alpha}{2}\right)+\left(\beta_{3} c_{440} \varepsilon_{110}+\beta_{2} e_{150}^{2}\right)\left(B_{13} \sin \frac{\alpha}{2}-B_{14} \cos \frac{\alpha}{2}\right)\right] \\
& A_{41}=B_{41}+B_{42} \cot 2 \alpha \\
& A_{42}=B_{43}+B_{44} \cot 2 \alpha \\
& C_{11}=B_{11}-B_{12} \cot \frac{\alpha}{2} \\
& C_{12}=B_{13}-B_{14} \cot \frac{\alpha}{2} \\
& C_{21}=B_{21}+B_{22} \cot \alpha \\
& C_{22}=B_{23}+B_{24} \cot \alpha \\
& C_{31}=B_{31}-B_{32} \cot \frac{3 \alpha}{2}-\frac{1}{12\left(c_{440} \varepsilon_{110}+e_{150}^{2}\right) \sin \frac{3 \alpha}{2}}\left[\left(\beta_{1} c_{440} \varepsilon_{110}+\beta_{2} e_{150}^{2}\right)\right. \text {. } \\
& \left.\left(B_{11} \cos \frac{\alpha}{2}+B_{12} \sin \frac{\alpha}{2}\right)+\left(\beta_{2}-\beta_{3}\right) e_{150} \varepsilon_{110}\left(B_{13} \cos \frac{\alpha}{2}+B_{14} \sin \frac{\alpha}{2}\right)\right] \\
& C_{32}=B_{33}-B_{34} \cot \frac{3 \alpha}{2}-\frac{1}{12\left(c_{440} \varepsilon_{110}+e_{150}^{2}\right) \sin \frac{3 \alpha}{2}}\left[( \beta _ { 1 } - \beta _ { 2 } ) c _ { 4 4 0 } e _ { 1 5 0 } \left(B_{11}\right.\right. \text {. } \\
& \left.\left.\cos \frac{\alpha}{2}+B_{12} \sin \frac{\alpha}{2}\right)+\left(\beta_{3} c_{440} \varepsilon_{110}+\beta_{2} e_{150}^{2}\right)\left(B_{13} \cos \frac{\alpha}{2}+B_{14} \sin \frac{\alpha}{2}\right)\right] \\
& C_{41}=B_{41}+B_{42} \cot 2 \alpha \\
& C_{42}=B_{43}+B_{44} \cot 2 \alpha
\end{aligned}
$$

Substituting Eq.(12)-(27) and (5)-(9) into Eq.(4), the displacement component and the electric potentialfor the I, II and III regions are obtained. The higher order stress and electric displacement fields will be given based on Eq.(10) finally. Then we the stress intensity factor (SIF) and electric displacement intensity factor (EDIF) can be expressed as follow

$$
\left\{\begin{array}{l}
K^{T}=\lim _{r \rightarrow 0} \sqrt{2 \pi r} \sigma_{y z}^{(1)}(r, 0)=\frac{\sqrt{2 \pi}}{2}\left(c_{4444}^{(1)} A_{11}^{(1)}-e_{15}^{(1)} B_{11}^{(1)}\right) \\
K^{D}=\lim _{r \rightarrow 0} \sqrt{2 \pi r} D_{y}^{(1)}(r, 0)=\frac{\sqrt{2 \pi}}{2}\left(e_{15}^{(1)} A_{11}^{(1)}+\varepsilon_{11}^{(1)} B_{11}^{(1)}\right)
\end{array}\right.
$$




\section{Conclusion}

The higher order crack-tip fields for an arbitrarily oriented crack oblique to the interface between FGPMs and HPMs are obtained based on the eigen-expansion method. The effect of non-homogeneity and crack oriented reflects only in the higher order items. The results show that the intensity factors depend on the material properties and crack oriented. It is obvious that the crack tip fields and intensity factors obtained herein provide basic solutions to fracture problems of FGPMs in experimental analysis and engineering practice.

\section{Acknowledgements}

The research is supported by the National Natural Science Foundation of China (No11172332.)

\section{References}

[1] C. Li and G.J. Weng: Journal of Applied Mechanics-Transactions of the Asme. Vol.69(2002), p.481

[2] Y.S. Ing and J.H. Chen: Theoretical and Applied Fracture Mechanics. Vol.63(2013), p. 40

[3] B. M. Singh, J. Rokne and R. S. Dhaliwal: European Journal of Mechanics. Vol.27(2008), p.346

[4] C.-H. Chue, C.-N. Yeh: Meccanica. Vol.46 (2010), p. 447

[5] X. Han and T. Wang: International Journal of Solids and Structures, Vol.36(1999), p.4183 